\title{
A Novel Method for Analyzing Silver Sediment With High Precision
}

\author{
R. S. Davis and V. E. Bower \\ Center for Absolute Physical Quantities, National Bureau of Standards, Washington, DC 20234 \\ October 25, 1978
}

\begin{abstract}
A technique has been devised which is sufficiently accurate to aid in an electrochemical determination of the Faraday constant using the silver coulometer. The technique is used to recover the silver residue which falls from the anode during operation of the silver coulometer. In contrast to previous efforts at recovery, which involved weighing of the silver residue, the method here described is to convert the silver atoms to ions and then to plate the silver onto a cathode held at constant potential with respect to a reference electrode. The current involved in the electrolysis is integrated electronically. An overall standard deviation of $5 \mu \mathrm{g}$ is achieved for samples ranging in size from $400 \mu \mathrm{g}$ to $1.8 \mathrm{mg}$.
\end{abstract}

Key words: Controlled potential; coulometry; electrochemical equivalent of silver; electrochemisty; Faraday; potentiostat; silver; silver analysis.

\section{Introduction}

As a part of our effort to measure the electrochemical equivalent of silver and hence the Faraday constant $[1],{ }^{1}$ we encountered a problem of determining the amount of finelydivided silver residue at the bottom of a beaker containing aqueous perchloric acid and silver perchlorate. The metallic silver could be expected to total from $100 \mu \mathrm{g}$ to $2 \mathrm{mg}$. An acceptable uncertainty to the overall Faraday experiment dictated that the residue of metallic silver powder from a single Faraday run be recovered to an accuracy of $5 \mu \mathrm{g}$.

Briefly, the electrochemical equivalent of silver is found by passing a known amount of current through an electrolytic cell whose anode is a bar of silver. The reaction $\mathrm{Ag} \rightarrow \mathrm{Ag}^{+}+$e occurs at the anode. The loss in mass of the anode divided by the total charge passed determines the electrochemical equivalent. In practice, however, some of the anode is lost through the mechanical separation of small particles of silver. These fall to the bottom of the anode beaker, whose anolyte is a solution of dilute perchloric acid and silver perchlorate. The residue of metallic silver must be analyzed and an appropriate correction made.

In previous experiments to determine the electrochemical equivalent of silver [2], the anode residue was found by filtering it onto sintered glass and weighing. The weighing of a glass filter to an accuracy of $5 \mu \mathrm{g}$, however, seemed to us overly arduous if not completely futile.

Instead we performed the analysis of silver residue to the needed accuracy using a controlled-potential coulometric technique. The silver was first dissolved in nitric acid.

\footnotetext{
${ }^{1}$ Figures in brackets indicate literature references at the end of this paper.
}

Evaporation then left a deposit of silver nitrate. After dissolution in supporting electrolyte, the resulting silver ion was plated onto a platinum electrode held at constant potential. The time integral of the current passed during the analysis was measured electronically. Details of this procedure are provided below.

\section{Apparatus}

The three-electrode potentiostat built for this work follows the ideas first put forth by Booman [3]. In detailed design, our apparatus closely resembles that of Jones et al. [4]. The device is shown schematically in figure 1. All three operational amplifiers are solid-state and chopper-stabilized with a maximum output current of $25 \mathrm{~mA}$. A limiting resistor (not shown) in the circuit assures that the maximum current will not exceed $9 \mathrm{~mA}$. Amplifier 1 is provided with an overload recovery circuit (not shown) suggested by the manufacturer, which prevents it from saturating. Without this external circuit, it would take up to $3 \mathrm{~s}$ for the amplifier to recover from saturation, leading to an error of as much as $30 \mu \mathrm{g}$ in the silver determination.

The integrating capacitor was $10 \mu \mathrm{f}$ with polystyrene dielectric. The integrator was calibrated in coulombs by running the apparatus in a constant current mode: reference and anode leads were shorted and a calibrated resistor $\left(R_{s}\right.$ in fig. 1) was placed between this junction and the cathode lead. In this way, a constant current of $E_{v a r} / R_{s}$ was produced by Amplifier 1 when the apparatus was turned on. The actual current was accurately known by reading the voltage across $R_{s}$ with a high-impedance voltmeter. The length of time the current ran could be determined with sufficient accuracy 


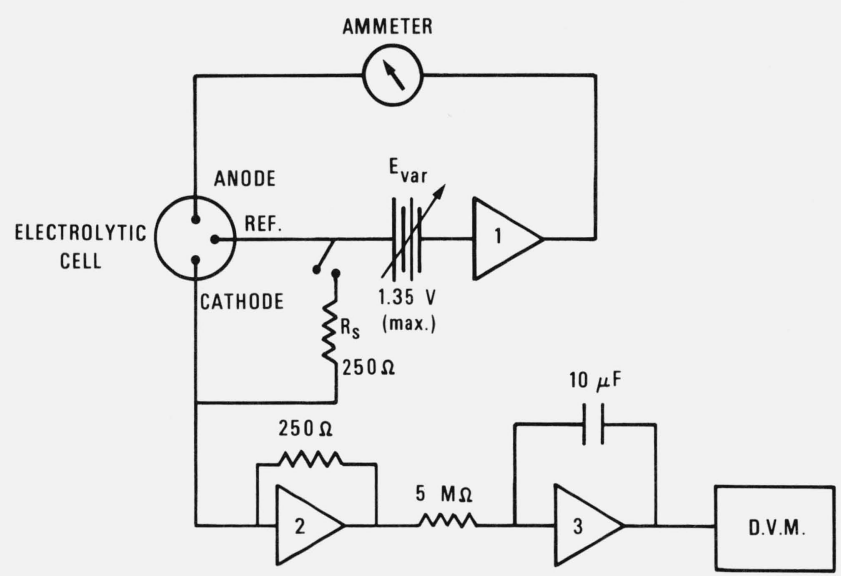

FiguRE 1. Schematic representation of electronics used in silver analysis. Amplifier 1 forces the cathode to have a potential difference of $E_{v a r}$ vs. the reference electrode. Amplifier 2 converts the current passing through the cell to a voltage. This voltage drops to virtual ground across the $5 \mathrm{M} \Omega$ resistor and the resulting current is integrated by Amplifier 3 . The integrator can be calibrated by disconnecting the cell, shorting the anode and reference leads, and switching in $R_{s}$

using a stopwatch. The final voltage on the integrating capacitor was read using the same high-impedance voltmeter. Thus the capacitor voltage could be related to the total charge passed by Amplifier 1 while current was flowing. By varying $E_{\text {var }}$ and $t$, the time current flowed, we discovered that the integrating capacitor had negligible voltage dependence and leakage current. By "negligible" it is meant that no corrections needed to be applied in order to achieve the desired accuracy for the silver analysis.

Monitoring of the capacitor voltage by a strip-chart recorder was found to be useful both during calibration at constant current and during constant potential analyses. Recorder traces are not as precise as voltmeter readings and hence are not used for taking data but they do give unambiguous evidence of many types of trouble.

During controlled-potential coulometric runs, current was monitored by an ammeter placed in series with the test cell. The final current of a silver analysis usually dropped to a few microamperes. The actual number could be read to an uncertainty of $0.1 \mu \mathrm{A}$.

The test cell for silver analyses closely follows that of reference [4]. The cathode compartment is a modified $80 \mathrm{ml}$ weighing bottle. The modification consists of a glass sidearm connected to the weighing bottle near its bottom. The wall common to the bottle and arm is replaced with a sintered glass filter of fine porosity. The other end of the arm can be connected to additional glassware. By virtue of this design, the test cell also serves as anode compartment for the initial anodic dissolution of silver. (A special tube may be attached to the arm forming a salt bridge to the remainder of the coulometer). Upon conclusion of the anodic dissolution phase, the arm is attached to an aspirator and the anolyte drained off. All that remains is the residue of silver, which is trapped by the filter in the bottle wall. The bottle then becomes the cathode compartment for the reaction $\mathrm{Ag}^{+}+e \rightarrow \mathrm{Ag}$. The silver residue need never be transferred to a different vessel for analysis.

The top of the bottle is plugged with teflon during the analysis. An anode compartment, reference electrode compartment, gas dispersion tube and cathode are all held in the teflon plug. The cathode is a strip of platinum gauze $40 \mathrm{~mm}$ by $100 \mathrm{~mm}$. The two compartments are formed by glass tubes (11 mm I.D.) open at the top and terminated with sintered glass frits of fine porosity at the bottom. Electrolyte leaks through the frits but the rate is slow, amounting to several cubic centimeters during a silver analysis. A coil of platinum wire serves as the anode. A standard calomel electrode (S.C.E.) is used as the reference electrode.

A specially constructed termination for the side arm allows the arm to be pressurized with nitrogen gas to prevent catholyte from seeping out of the bottle and into the arm during analysis of silver residue.

A magnetic stirrer is used during electrolyses.

The supporting electrolyte was chosen simply by consulting the available literature [5]. No attempt to find other electrolytes was made. The supporting electrolyte is a solution of $0.8 \mathrm{M} \mathrm{NaNO}_{3}$ and $0.05 \mathrm{M} \mathrm{HClO}_{4}$. The chemicals are reagent grade. We did not attempt further purification.

\section{Experimental}

In order to test the silver analysis procedure, standard solutions of silver were prepared. Silver shot, said to be 99.999\% pure by the manufacturer, was weighed on a microbalance. A single pellet of $50 \mathrm{mg}$ was used for each bottle of standard solution. A one-liter glass bottle with glass stopper was then tared on an analytical balance. The silver pellet was placed in the bottle with $500 \mathrm{~cm}^{3}$ of $1 / 4 \mathrm{M} \mathrm{HNO}_{3}$. The bottle was heated until the pellet had completely dissolved in the acid. Upon cooling to room temperature, the bottle was reweighed. The concentration of silver ion in solution was thus known to 5 parts in 100,000 .

In using the standard solution to evaluate our silver determination procedure, the modified bottle was dispensed with. The cathode cell was simply an $80 \mathrm{ml}$ weighing bottle. An appropriate amount of standard solution was drawn from the stock by pipette and weighed in the previously tared weighing bottle. After weighing, the bottle was opened and the solution allowed to evaporate under a hood. The evaporation time was hastened by partially immersing the bottle in a bath of $50{ }^{\circ} \mathrm{C}$ distilled water. Just prior to complete evaporation, the bottle was removed from the bath and placed in a drying oven at a temperature well below the dissociation temperature of silver nitrate. Evaporation was deemed complete when no acid odor could be detected in the bottle. At this point, the bottle now contained a white spot at the bottom, which was a residue of silver nitrate. 
Originally, we allowed complete evaporation to occur while the bottle rested in the bath of hot water. Occasionally, however, the residual white spot was found to be circumscribed with a brownish substance. Final analysis invariably showed a deficit of silver ion recovered in these cases. We conclude that the brown color is caused by thermal dissociation of silver nitrate due to hot spots in the bath.

When the supporting electrolyte is added to the cathode compartment, the silver nitrate immediately dissolves leaving one in a position to determine a known amount of silver ion.

Choice of reduction potential is important to the success of the analysis. At the proper potential, all the silver will be recovered with a minimum of extraneous cathode reactions adding to the integrated current. The proper potential was chosen experimentally, following the suggestion of Lingane is his seminal paper of 1948 [6]. A sample containing $1 \mathrm{mg}$ of silver ion was prepared as described above. The ions were then reduced at the platinum cathode, whose potential was varied in steps relative to the S.C.E. The integrated electrical current (charge) passed could then be directly related to the amount of silver reduced at each cathode potential. The results, of course, follow Nernst's curve for a singly-charged ion. From the curve, we deduced that our working electrode potential should be held at $+0.12 \mathrm{~V}$ vs. S.C.E.. in order that less then $1 \mu \mathrm{g}$ of silver remain ionized at the end of a determination.

The current in a controlled-potential coulometric analysis falls exponentially to a steady background level. Our analyses were judged complete when this level, usually more than three orders of magnitude below the initial current, was reached as evidenced by observing a microammeter in series with the anode. The average duration of our analyses was 15 minutes from the time the potentiostat was turned on.

Ideally the background current is negligible. In actuality this is rarely the case. The sources of background current have been discussed in detail by Meites and Moros [7]. It is usual to correct for this current, as we did, by assuming it to have been constant throughout the titration. At best, this correction imposes a limit on precision and at worst, as Meites and Moros point out, a limit on accuracy. In the case of silver analyses, the background current is extremely sensitive to the presence of dissolved oxygen gas in the electrolyte. The particular case of oxygen has been examined in reference [4]. In our analyses, pure nitrogen gas was bubbled vigorously through the catholyte by means of the gas dispersion tube of our apparatus. For best results, the bubbling was begun 10 minutes prior to the start of an analysis and continued until the analysis ended.

In all, 17 analyses were made. Samples were drawn from three different stock solutions of varying concentration. The background current was noted at the end of the titration and a correction was made assuming this current was constant throughout the electrolysis. Just before a measurement, the platinum cathode was soaked in hot nitric acid and rinsed with distilled water. The cathode was then dipped into hot aqua regia, rinsed with distilled water and heated to incandescence in the oxyidizing flame of a propane torch. Between titrations the glassware was leached with hot nitric acid, rinsed with distilled water, washed with detergent, rinsed again with distilled water and finally dried in an oven.

The results of the 17 determinations are shown in table I where the measured total charge has been converted to a mass of silver by using the value for the electrochemical equivalent of silver.

TABLE I.

Comparison of amount of silver recovered with amount present. Electrical current passing through the cell at the completion of the analysis is shown in the last column.

\begin{tabular}{c|c|c|c}
\hline \hline & Silver, $\mu \mathrm{g}$ & & \multirow{2}{*}{$\begin{array}{c}\text { Background } \\
\text { Current, } \mu \mathrm{A}\end{array}$} \\
\cline { 1 - 3 } Present & Found & Error & 5.0 \\
$1,793.7$ & $1,794.3$ & +0.6 & 3.3 \\
$1,794.1$ & $1,791.8$ & -2.3 & 4.4 \\
$1,794.0$ & $1,796.9$ & +2.9 & 4.5 \\
$1,794.4$ & $1,795.4$ & +1.0 & 1.6 \\
$1,800.5$ & $1,803.3$ & +2.8 & 1.5 \\
875.0 & 886.1 & +11.1 & 1.4 \\
874.7 & 882.4 & +7.7 & 2.0 \\
875.1 & 885.1 & +10.6 & 7.5 \\
874.5 & 886.9 & +12.4 & 3.1 \\
875.5 & 879.3 & +3.8 & 5.9 \\
875.5 & 874.8 & -0.7 & 2.4 \\
411.3 & 421.5 & +10.2 & 1.6 \\
411.8 & 419.3 & +7.5 & 3.2 \\
411.9 & 412.5 & +0.6 & 4.2 \\
412.1 & 418.4 & +6.3 & 3.8 \\
411.8 & 410.4 & -0.6 & 8.0 \\
412.3 & 421.4 & +9.1 & \\
\multicolumn{3}{|c}{ Average error: $+4.9 \mu \mathrm{g}$} \\
\hline \multicolumn{3}{|c}{ Standard deviation: $\pm 4.8 \mu \mathrm{g}$} \\
\hline
\end{tabular}

\section{Discussion}

This analytical technique was developed not simply to determine the amount of silver powder at the bottom of a beaker but to apply this determination as part of a measurement of the electrochemical equivalent of silver. Hence the percentage error of silver recovered does not concern us as much as does the actual amount. From table I it is observed that, on the average, an excess of $4.4 \times 10^{-3}$ coulombs is detected. This leads to an average error of $4.9 \mu \mathrm{g}$ of silver with a standard deviation of a single measurement of $4.8 \mu \mathrm{g}$.

A curious result is that the errors in the $1,800 \mu \mathrm{g}$ analyses seem to have a different distribution from those of the 875 $\mu \mathrm{g}$ and $412 \mu \mathrm{g}$ analyses. In fact, the largest samples appear to have both the smallest error and the smallest range of error. The reason behind this behavior might reside in a combination of the time taken for the platinum cathode to 
become a completely silver cathode during the silver reduction and the different rates of extraneous reactions occurring at platinum and silver cathodes. Exactly the same consideration might explain the positive average error of table 1. It was found, for instance, that the potentiometric analysis of a blank (i.e. a sample containing no silver) always produced errors much larger than any encountered with samples of known, finite amount of silver.

Instead of pursuing the cause of the small observed error anomaly, we chose simply to account for any size effects by running a known sample of approximately the same amount as the unknown residue and applying the known result as one would normally apply a blank correction.

The above-described analytical procedure is able to provide the required accuracy for use as part of our determination of the electrochemical equivalent of silver. Aside from yielding the requisite accuracy, however, the procedure also recommends itself by virtue of its ease relative to other analytical techniques. The manipulations require little in the way of delicacy or analytical training. It is quite possible that this technique may yet be improved by more attention to the choices of cathode material and supporting electrolyte, as well as to cell design.

A final point deserves mention. It has been emphasized that this procedure is designed for use in the measurement of the electrochemical equivalent of silver. However, the electrochemical equivalent of silver was in turn used to obtain the data of table I. A possible logical fallacy is easily avoided. In the overall measurement of the electrochemical equivalent, it is not necessary to know the mass of the silver residue. The total charge of this residue when converted to singly ionized silver suffices. It is just this quantity which our procedure measures directly.

\section{References}

[1] Bower, V. E. and Davis, R. S., A New Determination of the Faraday by means of the silver coulometer, Atomic Masses and Fundamental Constants 5, J. H. Sanders and A. H. Wapstra, eds., Plenum Press, N. Y., 578-583 (1975).

[2] Craig, D. N., Hoffman, J. E., Law, C. A., and Hamer, W. J., Determination of the value of the Faraday with a silver-perchloric acid coulometer, J. Res. Nat. Bur. Stand. (U.S.) 64A (Phys. and Chem.) No. 5, 381-402 (1960).

[3] Booman, G. L., Instrument for controlled-potential electrolysis and precision coulometric integration, Anal. Chem. 29, No. 2, 213218 (1957).

[4] Jones, H. C., Shults, W. D., and Dale, J. M., High-sensitivity controlled potential coulometric titrator, Anal. Chem. 37, No. 6, 680-687 (1965).

[5] Merritt, Jr. L. L., Martin, Jr. L. L., and Bedi, R. D., Apparatus for automatic controlled potential electrolysis using an electronic coulometer, Anal. Chem. 30, No. 4, 487-492 (1958).

[6] Lingane, J. J., Controlled potential electroanalysis, Analyti. Chim. Acta 2, 584-601 (1948).

[7] Meites, L. and Moros, S., Background corrections in controlledpotential coulometric analysis, Anal. Chem., 31, No. 1, 23-28 (1959). 